\title{
AN APPROXIMATION PROPERTY OF IMPORTANCE IN INVERSE SCATTERING THEORY
}

\author{
DAVID COLTON ${ }^{1}$ AND BRIAN D. SLEEMAN ${ }^{2}$ \\ ${ }^{1}$ Department of Mathematical Sciences, University of Delaware, \\ Newark, DE 19716, USA \\ ${ }^{2}$ School of Mathematics, University of Leeds, Leeds LS2 9JT, UK
}

(Received 30 May 2000)

\begin{abstract}
A key step in establishing the validity of the linear sampling method of determining an unknown scattering obstacle $D$ from a knowledge of its far-field pattern is to prove that solutions of the Helmholtz equation in $D$ can be approximated in $H^{1}(D)$ by Herglotz wave functions.

To this end we establish the important property that the set of Herglotz wave functions is dense in the space of solutions of the Helmholtz equation with respect to the Sobolev space $H^{1}(D)$ norm.
\end{abstract}

Keywords: inverse obstacle scattering; Herglotz wave functions; the linear sampling method

AMS 2000 Mathematics subject classification: Primary 35R30

Secondary 35P25

\section{Introduction}

This paper is devoted to the problem of deriving an approximation theorem for the Helmholtz equation which is of central importance in the linear sampling method for solving the inverse scattering problem for time-harmonic acoustic waves [5]. In the explanation and solution of the problem in question we will, for the sake of simplicity, restrict ourselves to two-dimensional problems. However, a major point of our analysis is that it remains valid for the three-dimensional case.

The scattering of a time-harmonic acoustic plane wave by a bounded obstacle $D$ leads to the problem of determining a scattered field $u^{\mathrm{s}}$ which satisfies the Helmholtz equation and Sommerfield radiation condition in the exterior of $D$ subject to certain boundary conditions for $u^{\mathrm{s}}$ on the boundary $\partial D$ of $D[\mathbf{2}]$. In particular, if $D$ is sound hard, $u^{\mathrm{s}}$ satisfies a Neumann boundary condition on $\partial D$ and if $D$ is penetrable (and possibly inhomogeneous), then $u^{\mathrm{s}}$ satisfies a transmission boundary condition. In either case it can be shown [2] that, for $x \in R^{2}, u^{\mathrm{s}}(x)$ has the asymptotic behaviour

$$
u^{\mathrm{s}}(x)=\frac{\mathrm{e}^{\mathrm{i} k r}}{\sqrt{r}} u_{\infty}(\hat{x}, d)+O\left(r^{-3 / 2}\right),
$$

where $r=|x|, \hat{x}=x /|x|, k>0$ is the wavenumber and $d$ is a unit vector in the direction of propagation of the incident plane wave. The inverse scattering problem of interest to 
us is the problem of determining $D$ from a knowledge of the far-field pattern $u_{\infty}(\hat{x}, d)$ for $\hat{x}$ and $d$ on the unit circle $\Omega$, where it is not known a priori whether or not $D$ is sound hard or penetrable.

A recently developed method for solving this inverse scattering problem is the linear sampling method $[\mathbf{1}, \mathbf{3}-\mathbf{5}]$. In this method, a parameter $y \in R^{2}$ is introduced and, for each fixed $y$, a solution $g=g(\cdot, y) \in L^{2}(\Omega)$ is sought to the far-field equation

$$
\int_{\Omega} u_{\infty}(\hat{x}, d) g(d, y) \mathrm{d} s(d)=\mathrm{e}^{-\mathrm{i} k \hat{x} \cdot y} .
$$

It is then shown in the above-referenced papers that $\partial D$ is determined as the locus of points where $\|g(\cdot, y)\|_{L^{2}(\Omega)}$ tends to infinity as a function of the parameter point $y$. A key step in establishing the validity of the linear sampling method is to show that solutions of the Helmholtz equation in $D$ can be approximated in $C^{1}(\bar{D})$ by Herglotz wave functions, i.e. solutions of the Helmholtz equation of the form

$$
v_{g}(x):=\int_{\Omega} \mathrm{e}^{\mathrm{i} k x \cdot d} g(d) \mathrm{d} s(d),
$$

where $g \in L^{2}(\Omega)$.

To be more specific, define the fundamental solution to the Helmholtz equation by

$$
\Phi(x, y):=\frac{1}{4} \mathrm{i} H_{0}^{(1)}(k|x-y|),
$$

where $H_{0}^{(1)}$ denotes a Hankel function of the first kind of order zero. Then, in the case of a sound-hard scatterer and assuming $k^{2}$ is not a Neuman eigenvalue, it is desired to approximate the solution $u$ of

$$
\left.\begin{array}{ll}
\Delta_{2} u+k^{2} u=0, & \text { in } D, \\
\frac{\partial u}{\partial \nu}=\frac{\partial \Phi}{\partial \nu}(\cdot, y), & \text { on } \partial D,
\end{array}\right\}
$$

in $C^{1}(\bar{D})$ by a Herglotz wave function $v_{g}$, where, in (1.5), $\nu$ denotes the unit outward normal to $\partial D$. As $y$ tends to $\partial D$ it is seen $[\mathbf{1}, \mathbf{4}]$ that $v_{g}$ becomes unbounded and hence so does the kernel $g=g(\cdot, y)$. It is then shown that (up to a constant factor) $g$ can be determined as the (approximate) solution of the far-field equation (1.2). Similar considerations apply to the case of a penetrable scatterer [3]. A short examination of the above-referenced papers shows that since the fundamental solution $\Phi=\Phi(\cdot, y)$ is not in the Sobolev space $H^{1}(D)$ for $y \in \partial D$, it suffices to show that the solution $u$ of (1.5) can be approximated by a Herglotz wave function in $H^{1}(D)$.

In $[\mathbf{1}],[\mathbf{3}]$ and $[\mathbf{4}]$, the above approximation property was obtained by referring to the results of Vekua [9]. However, these results are only valid in $R^{2}$ and the techniques used cannot be extended to the three-dimensional case. The only corresponding approximation result in $R^{3}$ is due to Ochs [8], who needed to assume that $D$ is star-like with respect to the origin. Since this last assumption is clearly not satisfactory from the point of view of inverse scattering, it is important to establish the denseness of Herglotz wave functions in 
$H^{1}(D)$ in a manner that is valid in both two and three dimensions, thus guaranteeing the validity of the linear sampling method in both of these cases. The purpose of this paper is to do this. As already mentioned, for the sake of simplicity we will carry out our analysis in $R^{2}$, noting that our analysis has an immediate and straightforward generalization to the three-dimensional case. We will base our analysis on potential theory and the method of integral equations, although it is clear that at certain points we could also have appealed to the general theory of elliptic partial differential equations.

\section{An approximation theorem}

Let $D$ be a bounded domain in the plane containing the origin such that $R^{2} \backslash \bar{D}$ is connected. We assume that $D$ has a $C^{2}$ boundary $\partial D$ and let $\nu$ denote the unit outward normal to $\partial D$. For $n=0, \pm 1, \pm 2, \ldots$, we define $u_{n}$ by

$$
u_{n}(x):=J_{n}(k r) \mathrm{e}^{\mathrm{i} n \theta},
$$

where $J_{n}$ is a Bessel function of order $n$ and $(r, \theta)$ are the polar coordinates of $x \in R^{2}$. It follows from (1.3) and the Jacobi-Anger expansion [2, p. 67] that if $\left\{a_{n}\right\} \in \ell^{2}$, then

$$
v(x):=\sum_{-\infty}^{\infty} a_{n} u_{n}(x)
$$

is a Herglotz wave function that satisfies the Helmholtz equation

$$
\Delta_{2} v+k^{2} v=0
$$

in all of $R^{2}$.

Theorem 2.1. The set

$$
\left\{\frac{\partial u_{n}}{\partial \nu}+\mathrm{i} u_{n}\right\}
$$

is complete in $H^{-1 / 2}(\partial D)$.

Proof. It suffices to show that if $g \in H^{1 / 2}(\partial D)$ and

$$
\int_{\partial D} g(y)\left(\frac{\partial}{\partial \nu}+\mathrm{i}\right) u_{n}(y) \mathrm{d} s(y)=0
$$

for $n=0, \pm 1, \pm 2, \ldots$, then $g=0$. Hence, suppose that (2.4) is valid for some $g \in$ $H^{1 / 2}(\partial D)$ and let $B$ be a disc centred at the origin and containing $D$ in its interior. Then, from the addition formula for Bessel functions and (2.4), we can conclude that if $\Phi$ is the fundamental solution defined by (1.4), then

$$
u(x):=\int_{\partial D} g(y)\left(\frac{\partial}{\partial \nu_{y}}+\mathrm{i}\right) \Phi(x, y) \mathrm{d} s(y)
$$

is identically zero for $x \in R^{2} \backslash \bar{B}$. By the analyticity of solutions to the Helmholtz equation, we can conclude that $u(x)=0$ for $x \in R^{2} \backslash \bar{D}$. 
If $g \in C(\partial D)$, we could now conclude from (2.5) that

$$
2 u(x)=g(x)+2 \int_{\partial D} g(y)\left(\frac{\partial}{\partial \nu_{y}}+\mathrm{i}\right) \Phi(x, y) \mathrm{d} s(y)
$$

for $x \in \partial D$. Using Theorems 8.20 and 8.24 of $[\mathbf{6}]$ and the trace theorem, we see that (2.6) is valid for $g \in H^{1 / 2}(\partial D)$, where $u(x), x \in \partial D$, is interpreted in terms of the trace theorem. In particular,

$$
0=g(x)+2 \int_{\partial D} g(y)\left(\frac{\partial}{\partial \nu_{y}}+\mathrm{i}\right) \Phi(x, y) \mathrm{d} s(y)
$$

for $x \in \partial D$. Hence $g=K g$, where $K$ is an integral operator with weakly singular kernel, and we can conclude that for $m$ sufficiently large, $g=K^{m} g$ is continuous.

Letting the subscripts \pm denote the limits as $x$ tends to $\partial D$ from outside and inside $D$, respectively, we can conclude from (2.5) and the continuity properties of double and single layer potentials $[6]$ that

$$
\left.\begin{array}{c}
u_{+}-u_{-}=g \\
\left(\frac{\partial u}{\partial \nu}\right)_{+}-\left(\frac{\partial u}{\partial \nu}\right)_{-}=-\mathrm{i} g \quad \text { on } \partial D,
\end{array}\right\}
$$

and, since

$$
u_{+}=\left(\frac{\partial u}{\partial \nu}\right)_{+}=0
$$

we have

$$
\left(\frac{\partial u}{\partial \nu}\right)_{-}+\mathrm{i} u_{-}=0 \quad \text { on } \partial D
$$

Hence $u$, as defined by (2.5), is a solution of the Helmholtz equation in $D$ and continuously assumes the boundary data (2.9) on $\partial D$. An elementary application of Green's theorem now implies that $u(x)=0$ for $x \in D$. Since we already know that $u(x)=0$ for $x \in R^{2} \backslash \bar{D}$, the first relation in (2.8) now implies that $g=0$, and the proof is finished.

Now consider the problem of finding a weak solution of the boundary-value problem

$$
\left.\begin{array}{rl}
\Delta_{2} u+k^{2} u=0, & \text { in } D, \\
\frac{\partial u}{\partial \nu}+\mathrm{i} u=f, & \text { on } \partial D,
\end{array}\right\}
$$

where $f \in H^{-1 / 2}(\partial D)$ (cf. $\S 8.3$ of $[6]$ ). The uniqueness of a weak solution to $(2.10)$ follows in a straightforward manner (cf. Theorem 8.19 of $[\mathbf{6}]$ ). We want to show that a solution exists and can be represented in the form of a single-layer potential with density $\phi \in H^{-1 / 2}(\partial D)$. To this end we look for a solution of (2.10) in the form

$$
u(x)=\int_{\partial D} \phi(y) \Phi(x, y) \mathrm{d} s(y),
$$


where $\phi \in H^{-1 / 2}(\partial D)$. Then, proceeding as in Theorem 8.26 of $[6]$, we see that (2.11) will be a solution of (2.10) provided that

$$
2 f=\phi+K \phi
$$

where

$$
K \phi(x):=2 \int_{\partial D} \phi(y)\left(\frac{\partial}{\partial \nu_{x}}+\mathrm{i}\right) \Phi(x, y) \mathrm{d} s(y)
$$

for $x \in \partial D$.

To show the existence of a solution $\phi \in H^{-1 / 2}(\partial D)$ to (2.12), by the Riesz theory and Theorem 8.20 of [6], we need to show that if $\phi \in H^{-1 / 2}(\partial D)$ is a solution of the homogeneous equation $\phi+K \phi=0$, then $\phi=0$. To this end, if $\phi+K \phi=0$, then, as in Theorem 2.1, we can conclude that $\phi \in C(\partial D)$, and hence $u$, as defined by (2.11), is a classical solution of (2.10) for $f=0$, and hence $u(x)=0$ for $x \in D$. For $x \in R^{2} \backslash \bar{D}$ we now see that $u$ is a radiating solution of the Helmholtz equation vanishing on $\partial D$, and hence $u(x)=0$ for $x \in R^{2} \backslash \bar{D}$ (Theorem 3.7 of [2]). By the continuity properties of single-layer potentials $[\mathbf{6}]$ we can now conclude that $u_{+}-u_{-}=\phi=0$ and we are done.

Theorem 2.2. There exists a positive constant $C$ such that if $u$ is the unique weak solution of (2.10), then

$$
\|u\|_{H^{1}(D)} \leqslant C\|f\|_{H^{-1 / 2}(\partial D)} .
$$

Proof. This follows from the fact that $u$ can be represented as a single-layer potential and Theorem 8.24 of $[\mathbf{6}]$, noting that the singular part of $\Phi$ is the fundamental solution for Laplace's equation.

Theorem 2.2 could also be obtained by using variational methods for elliptic boundaryvalue problems. However, as mentioned in $\S 1$, we prefer to base all of our analysis on potential theory, rather than appealing to more general results.

Theorems 2.1 and 2.2 now yield our desired approximation result, where in the theorem the normal derivative of $u$ is in $H^{-1 / 2}(\partial D)$, since $u \in H^{1}(D)$ is a solution of the Helmholtz equation [7].

Theorem 2.3. With respect to the $H^{1}(D)$ norm, the set of Herglotz wave functions is dense in the space of solutions to the Helmholtz equation.

Proof. Let $u \in H^{1}(D)$ be a solution of the Helmholtz equation in $D$. Then by the trace theorem and the above comment,

$$
f=\frac{\partial u}{\partial \nu}+\mathrm{i} u \in H^{-1 / 2}(\partial D)
$$

and, by Theorem 2.1, $f$ can be approximated in $H^{-1 / 2}(\partial D)$ by $(\partial v / \partial \nu)+\mathrm{i} v$, where $v$ is a Herglotz wave function. The theorem now follows from Theorem 2.2.

Acknowledgements. The research of D.C. was supported in part by a grant from the US Air Force Office of Scientific Research. This paper was written while D.C. was 
visiting B.D.S. at the University of Leeds, supported by grants from the London Mathematical Society, the Edinburgh Mathematical Society and The Royal Society of Edinburgh. D.C. gratefully acknowledges the hospitality of both B.D.S. and the School of Mathematics at the University of Leeds. Finally, both authors would like to acknowledge the hospitality of the Victoria pub in Leeds where the ideas for this paper were discussed in a congenial atmosphere.

\section{References}

1. D. Colton And A. Kirsch, A simple method for solving inverse scattering problems in the resonance region, Inverse Problems 12 (1996), 383-393.

2. D. Colton And R. KRess, Inverse acoustic and electromagnetic scattering theory, 2nd edn (Springer, Berlin, 1998).

3. D. Colton and M. Piana, The simple method for solving the electromagnetic inverse scattering problem: the case of TE polarized waves, Inverse Problems 14 (1998), 597-614.

4. D. Colton, M. Piana And R. Potthast, A simple method using Morozov's discrepancy principle for solving inverse scattering problems, Inverse Problems 13 (1997), 1477-1493.

5. D. Colton, J. CoYle And P. Monk, Recent developments in inverse acoustic scattering theory, SIAM Rev. 42 (2000), 369-414.

6. R. KRESS, Linear integral equations, 2nd edn (Springer, New York, 1999).

7. W. MCLEAN, Strongly elliptic systems and boundary integral equations (Cambridge University Press, 2000).

8. R. OCHS, A version of Runge's theorem for the Helmholtz equation with applications to scattering theory, Proc. Edinb. Math. Soc. 32 (1989), 107-119.

9. I. N. VeKUA, New methods for solving elliptic equations (North Holland, Amsterdam, 1967). 